\title{
A numerical method for the expected penalty-reward function in a Markov-modulated jump-diffusion process
}

\author{
Peter Diko*, Miguel Usábel \\ Department of Business Administration, Universidad Carlos III de Madrid, Av. Universidad Carlos III 22, 28270 Colmenarejo, Madrid, Spain
}

\section{JEL classification:}

G22

Keywords:

Expected penalty-reward function

Markov-modulated process

Jump-diffusion process

Volterra integro-differential system of equations

Subject Category and Insurance Branch

Category:

IM11

IM13

\begin{abstract}
A B S T R A C T
A generalization of the Cramér-Lundberg risk model perturbed by a diffusion is proposed. Aggregate claims of an insurer follow a compound Poisson process and premiums are collected at a constant rate with additional random fluctuation. The insurer is allowed to invest the surplus into a risky asset with volatility dependent on the level of the investment, which permits the incorporation of rational investment strategies as proposed by Berk and Green (2004). The return on investment is modulated by a Markov process which generalizes previously studied settings for the evolution of the interest rate in time. The Gerber-Shiu expected penalty-reward function is studied in this context, including ruin probabilities (a first-passage problem) as a special case. The second order integro-differential system of equations that characterizes the function of interest is obtained. As a closed-form solution does not exist, a numerical procedure based on the Chebyshev polynomial approximation through a collocation method is proposed. Finally, some examples illustrating the procedure are presented.
\end{abstract}

\section{Introduction}

The risk process presented by Gerber (1970) extends the classical model of risk theory introducing a Brownian diffusion. The total claims follow a compound Poisson process $\left\{X_{t}, t \geq 0\right\}$ with Lévy measure $\lambda f(x) \mathrm{d} x, \lambda$ being the intensity of arrivals and $f$ the density of jumps. The collection of premiums is driven by a Wiener process $W_{t}^{c}$ independent of $X_{t}$ with drift $c$ and volatility $\sigma$, thus the perturbed risk process with initial surplus $u$ is given by

$$
\mathrm{d} R_{t}=c \mathrm{~d} t+\sigma \mathrm{d} W_{t}^{c}-\mathrm{d} X_{t}, \quad R_{0}=u .
$$

This process has been considered by Dufresne and Gerber (1991) where a defective renewal equation was derived for the probability of ruin $\psi(u)=\operatorname{Pr}(\tau<\infty)$ where $\tau=\inf \left\{t \geq 0: R_{t}<0\right\}$. A review of the research on this type of process can be found in Asmussen and Albrecher (2010), Chapter 11. Generalizations of the model are treated in Li and Garrido (2005), Sarkar and Sen (2005), and Morales (2007), whereas Ren (2005) gives explicit

\footnotetext{
* Corresponding author. Tel.: +34 918561254; fax: +34 916249607. E-mail addresses: Peter.Diko@uc3m.es (P. Diko), usabel@emp.uc3m.es (M. Usábel).
}

formulae to calculate the ruin probability and related quantities for phase-type distributed claims.

Let us now allow the insurer to invest the reserves $U_{t}$ into an asset with time-dependent Markov-modulated return rate (drift) $\Delta_{t}$ and volatility $\kappa\left(U_{t}\right)$, that possibly depends on the amount invested $U_{t}$, driven by a Wiener process $W_{t}^{I}$ independent of the risk process $R_{t}$

$\mathrm{d} U_{t}=\left(\Delta_{t} \mathrm{~d} t+\kappa\left(U_{t}\right) \mathrm{d} W_{t}^{I}\right) U_{t}+d R_{t}, \quad U_{0}=R_{0}=u$.

The drift parameter $\Delta_{t}$ is governed by a finite state homogeneous Markov process with state space $\left\{\delta_{1}, \ldots, \delta_{n}\right\}$, intensity matrix $Q=\left(q_{i j}\right)_{n \times n}$ and initial state $\delta_{i}$. For example, $\Delta_{t}$ can be used to model the risk free rate announced by a central bank that evolves according to the Markov process by, for instance, 25 basis point jumps. The state space would be in this case e.g.,

$1.00 \%, 1.25 \%, 1.50 \%, 1.75 \%, 2.00 \%, \ldots, 9.00 \%$.

This environment offers considerable versatility in capturing the evolution of interest rates since any diffusion model to forecast the yield curve can be approximated arbitrarily well by continuous time Markov chains; see Kushner and Dupuis (1992). Variation of the volatility according to the size of the funds invested is justified, for example, by Berk and Green (2004) as an implication of their study of the performance of mutual funds and resulting rational 
capital flows. A particular shape of $\kappa$ suggested in the cited paper, $\kappa(u)=\frac{\sigma_{r}}{\sqrt{u}}$, yields a surplus process in the form of an affine diffusion that was studied by Avram and Usabel (2008) in this context. Many practical ideas support a fund-dependent volatility, for instance the possibility to obtain more efficient portfolios, due to transaction costs, when more money is available. Model (2) is a generalization of the process considered most frequently in the literature where the return rate and the volatility are constant in time, $\Delta_{t}=\delta, \kappa(\cdot)=\sigma_{r}$, like in Paulsen (1993), Paulsen and Gjessing (1997), Wang (2001), Ma and Sun (2003), Gaier and Grandits (2004), Grandits (2005), Cai and Yang (2005) and Wang and $\mathrm{Wu}(2008)$.

The stochastic differential equation (2) can be arranged into

$\mathrm{d} U_{t}=\left(c+\Delta_{t} U_{t}\right) \mathrm{d} t+\sqrt{\sigma^{2}+\kappa^{2}\left(U_{t}\right) U_{t}^{2}} \mathrm{~d} W_{t}-\mathrm{d} X_{t}$

with initial condition $\left(U_{0}, \Delta_{0}\right)=\left(u, \delta_{i}\right)$. The expected penalty-reward function (see Gerber and Landry (1998)) is introduced

$\phi_{t}^{i}(u)=\mathrm{E}\left[\pi\left(U_{\tau}\right) \mathbb{I}(\tau \leq t)+P\left(U_{t}\right) \mathbb{I}(\tau>t) \mid U_{0}=u, \Delta_{0}=\delta_{i}\right]$

where $\tau=\inf \left\{s \geq 0: U_{s}<0\right\}$. If ruin occurs before the time horizon $t$, the penalty $\pi\left(U_{\tau}\right)$ applies to the overshoot $U_{\tau}$ at the ruin. Otherwise, the reward function $P\left(U_{t}\right)$ applies to the reserves at time $t$. The concept of the expected penalty-reward function presented in Gerber and Shiu (1997) and Gerber and Shiu (1998) is a quite general framework comprising several quantities of interest as a special case, such as the time to ruin, the amount at and immediately prior to ruin or survival probabilities.

For further analysis the smoothed version of the function $\phi_{t}^{i}(u)$ will be considered, namely its Laplace-Carson transform in time defined as

$\Upsilon_{\alpha}^{i}(u)=\int_{0}^{\infty} \alpha \mathrm{e}^{-\alpha t} \phi_{t}^{i}(u) \mathrm{d} t$.

Further, letting $H_{\alpha}$ be an exponentially distributed random variable with parameter $\alpha$, the former expression may be viewed as a penalty-reward function with an exponentially killed time horizon (see expression (6) in Avram and Usabel (2008))

$$
\begin{aligned}
\Upsilon_{\alpha}^{i}(u)= & \int_{0}^{\infty} \alpha \mathrm{e}^{-\alpha t} \phi_{t}^{i}(u) \mathrm{d} t=\mathrm{E}\left(\phi_{H_{\alpha}}^{i}(u)\right) \\
= & \mathrm{E}\left(\pi\left(U_{\tau}\right) \mathbb{I}\left(\tau \leq H_{\alpha}\right)\right. \\
& \left.+P\left(U_{H_{\alpha}}\right) \mathbb{I}\left(\tau>H_{\alpha}\right) \mid U_{0}=u, \Delta_{0}=\delta_{i}\right)
\end{aligned}
$$

where the last equality comes from substituting the definition of $\phi_{t}^{i}(u)$, in (4).

The function $\Upsilon_{\alpha}^{i}(u)$ is analytically more tractable than the original function while, at the same time, retains a probabilistic interpretation as a penalty-reward function considering an exponential random time horizon $H_{\alpha}$.

The results in this paper are organized as follows: in Section 2 an integro-differential system that characterizes the function of interest $\Upsilon_{\alpha}^{i}(u)$ is derived and the existence of the solution discussed. In Section 3 a numerical method to approximate the solution of the system via Chebyshev polynomials is considered and Section 4 offers some numerical illustrations.

\section{Integro-differential system}

This section presents further treatment of the transformed expected penalty-reward function defined by (5). The function $\Upsilon_{\alpha}^{i}(u)$ is dependent on the initial reserves $U_{0}=u$ and the starting return rate $\Delta_{0}=\delta_{i}$. Since the process driving the return rate $\Delta_{t}$ has a finite state space, the number of initial conditions is also finite. Therefore, one can consider the set of functions $\Upsilon_{\alpha}(u)=\left(\Upsilon_{\alpha}^{1}(u), \Upsilon_{\alpha}^{2}(u), \ldots, \Upsilon_{\alpha}^{n}(u)\right)$, each corresponding to different starting return rate from the state space $\left\{\delta_{1}, \ldots, \delta_{n}\right\}$. Below, a Volterra integro-differential system of equations for the functions $\Upsilon_{\alpha}^{1}(u), \Upsilon_{\alpha}^{2}(u), \ldots, \Upsilon_{\alpha}^{n}(u)$ is derived and, applying the result of Le and Pascali (2009), sufficient conditions for the existence of the solution are established.

Theorem 2.1. For all $\alpha \geq 0$, functions $\Upsilon_{\alpha}^{i}:[0, \infty) \rightarrow \mathbb{R}$ defined in (5) satisfy the following system of integro-differential equations

For $i=1, \ldots, n$

$\frac{1}{2}\left(\sigma^{2}+u^{2} \kappa^{2}(u)\right) \frac{\mathrm{d}^{2}}{\mathrm{~d} u^{2}} \Upsilon_{\alpha}^{i}(u)+\left(c+\delta_{i} u\right) \frac{\mathrm{d}}{\mathrm{d} u} \Upsilon_{\alpha}^{i}(u)$

$+\sum_{j=1}^{n} q_{i j} \Upsilon_{\alpha}^{j}(u)-(\alpha+\lambda) \Upsilon_{\alpha}^{i}(u)$

$+\lambda \int_{0}^{u} \Upsilon_{\alpha}^{i}(u-x) f(x) \mathrm{d} x$

$+\alpha P(u)+\lambda \int_{u}^{\infty} \pi(u-x) f(x) \mathrm{d} x=0$.

Given that $\lim _{u \rightarrow \infty} P(u)$ exists, $\sigma>0$ and assuming positive security loading for the reserve process (2), the boundary conditions of the system are

$\Upsilon_{\alpha}^{i}(0)=\pi(0-)$

$\lim _{u \rightarrow \infty} \Upsilon_{\alpha}^{i}(u)=\lim _{u \rightarrow \infty} P(u) \equiv P(\infty)$.

Moreover, if $f \in C^{2}[0, \infty), P(u)$ and $\kappa(u)$ are continuous for $u \geq 0$ and $\pi(u)$ integrable, then the system of Eq. (6) has a solution $\Upsilon_{\alpha}^{\bar{i}} \in$ $C^{2}[0, \infty), i=1, \ldots, n$.

Proof. First, a straightforward application of Ito's lemma yields the infinitesimal generator of the process $U_{t}$, which applied to the functions $\phi_{t}^{i}(u), i=1, \ldots, n$ defined by (4), yields

$$
\begin{aligned}
\mathcal{A} \phi_{t}^{i}(u)= & \frac{1}{2}\left(\sigma^{2}+u^{2} \kappa^{2}(u)\right) \frac{\mathrm{d}^{2}}{\mathrm{~d} u^{2}} \phi_{t}^{i}(u) \\
& +\left(c+\delta_{i} u\right) \frac{\mathrm{d}}{\mathrm{d} u} \phi_{t}^{i}(u)+\sum_{j=1}^{n} q_{i j} \phi_{t}^{j}(u) \\
& +\lambda \int_{0}^{\infty}\left(\phi_{t}^{i}(u-x)-\phi_{t}^{i}(u)\right) f(x) \mathrm{d} x .
\end{aligned}
$$

Functions $\phi_{t}^{i}(u)$ satisfy the Fokker-Planck equation (see e.g. Risken (1996))

$\mathcal{A} \phi_{t}^{i}(u)-\frac{\partial \phi_{t}^{i}(u)}{\partial t}=0$

with boundary conditions

$\phi_{0}^{i}(u)=P(u) \quad u>0$

$\phi_{t}^{i}(u)=\pi(u) \quad u<0$ and $t \geq 0$

for each $i=1,2, \ldots, n$. Using (9b) the following holds

$$
\begin{aligned}
\int_{0}^{\infty} \phi_{t}^{i}(u-x) f(x) \mathrm{d} x= & \int_{0}^{u} \phi_{t}^{i}(u-x) f(x) \mathrm{d} x \\
& +\int_{u}^{\infty} \pi(u-x) f(x) \mathrm{d} x .
\end{aligned}
$$

Substituting the infinitesimal generator and (10) into the FokkerPlanck equation yields

$$
\frac{1}{2}\left(\sigma^{2}+u^{2} \kappa^{2}(u)\right) \frac{\mathrm{d}^{2}}{\mathrm{~d} u^{2}} \phi_{t}^{i}(u)+\left(c+\delta_{i} u\right) \frac{\mathrm{d}}{\mathrm{d} u} \phi_{t}^{i}(u)
$$




$$
\begin{aligned}
& +\sum_{j=1}^{n} q_{i j} \phi_{t}^{j}(u)-\lambda \phi_{t}^{i}(u)+\lambda \int_{0}^{u} \phi_{t}^{i}(u-x) f(x) \mathrm{d} x \\
& +\lambda \int_{u}^{\infty} \pi(u-x) f(x) \mathrm{d} x-\frac{\partial \phi_{t}^{i}(u)}{\partial t}=0 .
\end{aligned}
$$

The system (6) is obtained taking the Laplace-Carson transform with respect to $t$ on both sides and expanding the last term integrating by parts

$$
\begin{aligned}
\int_{0}^{\infty} \alpha \mathrm{e}^{-\alpha t} \frac{\partial \phi_{t}^{i}(u)}{\partial t} \mathrm{~d} t & =-\alpha P(u)+\alpha \int_{0}^{\infty} \alpha \mathrm{e}^{-\alpha t} \phi_{t}^{i}(u) \mathrm{d} t \\
& =-\alpha P(u)+\alpha \Upsilon_{\alpha}^{i}(u)
\end{aligned}
$$

where the first boundary condition (9a) of the Fokker-Planck equation was used.

Concerning the boundary conditions of the integro-differential system, when the initial reserves are 0 and $\sigma>0$, the presence of the Wiener fluctuation in premiums causes immediate crossing of 0 level; see for example the proof of Theorem 2.1 in Paulsen and Gjessing (1997). The second condition is the asymptotic case $u \rightarrow \infty$ when under the assumption of positive security loading $\lim _{u \rightarrow \infty} \Upsilon_{\alpha}^{i}(u)=\lim _{u \rightarrow \infty} P(u)<\infty$.

To prove the existence of the solution, an equivalent system will be considered. A change of variable is now introduced in the System (6), $h(v)=u$, where $h:[0,1] \rightarrow[0, \infty)$ is an arbitrary strictly monotone, twice continuously differentiable function. The system can now be written in terms of the functions $\Gamma_{\alpha}^{i}(v)=$ $\Upsilon_{\alpha}^{i}(h(v))$.

$$
\begin{aligned}
& \text { For } i=1, \ldots, n \\
& A(v) \frac{\mathrm{d}^{2}}{\mathrm{~d} v^{2}} \Gamma_{\alpha}^{i}(v)+B_{i}(v) \frac{\mathrm{d}}{\mathrm{d} v} \Gamma_{\alpha}^{i}(v)+\sum_{j=1}^{n} q_{i j} \Gamma_{\alpha}^{j}(v) \\
& -(\alpha+\lambda) \Gamma_{\alpha}^{i}(v)+\lambda \int_{0}^{v} \Gamma_{\alpha}^{i}(y) f(h(v)-h(y)) h^{\prime}(y) \mathrm{d} y \\
& +\lambda S(v)+\alpha P(h(v))=0
\end{aligned}
$$

where

$$
\begin{aligned}
& A(v)=\frac{\sigma^{2}+h^{2}(v) \kappa^{2}(h(v))}{2\left[h^{\prime}(v)\right]^{2}} \\
& B_{i}(v)=\frac{c+\delta_{i} h(v)}{h^{\prime}(v)}-\frac{\left[\sigma^{2}+h^{2}(v) \kappa^{2}(h(v))\right] h^{\prime \prime}(v)}{2\left[h^{\prime}(v)\right]^{3}} \\
& S(v)=\int_{v}^{1} \pi(h(v)-h(y)) f(h(y)) h^{\prime}(y) \mathrm{d} y
\end{aligned}
$$

with boundary conditions

$$
\begin{aligned}
& \Gamma_{\alpha}^{i}(0)=\pi(0-) \\
& \Gamma_{\alpha}^{i}(1)=\lim _{u \rightarrow \infty} P(u) .
\end{aligned}
$$

Here $h^{\prime}$ and $h^{\prime \prime}$ denote the first and the second derivative of function $h$. Finally, by integration

$$
\begin{aligned}
\Gamma_{\alpha}^{i}(s)= & \Gamma_{\alpha}^{i}(0)+\int_{0}^{s} \frac{h^{\prime}(v)}{B_{i}(v)}\left[H(v)-\lambda \int_{0}^{v} f(h(v)\right. \\
& \left.-h(y)) \frac{h^{\prime}(y)}{h^{\prime}(v)} \Gamma_{\alpha}^{i}(y) \mathrm{d} y\right] \mathrm{d} v \\
H(v)= & \frac{-1}{h^{\prime}(v)}\left[A(v) \frac{\mathrm{d}^{2}}{\mathrm{~d} v^{2}} \Gamma_{\alpha}^{i}(v)+\sum_{j=1}^{n} q_{i j} \Gamma_{\alpha}^{i}(v)\right. \\
& \left.-(\alpha+\lambda) \Gamma_{\alpha}^{i}(v)+\alpha P(h(v))+\lambda S(v)\right] .
\end{aligned}
$$

The existence of the solution $\Gamma_{\alpha}^{i} \in C^{2}[0,1]$ is guaranteed by Theorem 2 in Le and Pascali (2009), as $H(v)$ is a continuous function and $f(h(v)-h(y)) \frac{h^{\prime}(y)}{h^{\prime}(v)}$ is integrable. The integrability is immediate as $f$ is a density function and $\frac{h^{\prime}(y)}{h^{\prime}(v)}$ is a bounded function of $y$ on $[0, v]$ for all $v$. This implies that $\Upsilon_{\alpha}^{i}(u)=\Gamma_{\alpha}^{i}\left(h^{-1}(u)\right)$, a solution to (6), exists and $\Upsilon_{\alpha}^{i} \in C^{2}[0, \infty)$.

\section{Numerical solution}

The second order system of integro-differential equation (6) that characterizes the Laplace-Carson transform of the expected penalty-reward function (5) does not have an explicit solution. In Akyuz-Dascioglu and Sezer (2005) and Akyuz-Dascioglu (2007) a numerical method was proposed for fairly general families of Fredholm-Volterra integro-differential systems of higher order which include the system treated in this article as a special case. The authors approximate the solution to the system by shifted Chebyshev polynomials on the interval $[0,1]$. A collocation method is used to fit the Chebyshev expansion of the solution. In order to adapt the procedure to system (6), we need to transform the domain of the unknown functions $\Upsilon_{\alpha}^{i}$, as was done in the proof of Theorem 2.1, from the interval $[0, \infty)$ to $[0,1]$. First, the solution $\Gamma_{\alpha}^{i}$ of the transformed system is found and then, applying the inverse transform, the functions of interest $\Upsilon_{\alpha}^{i}$ are recovered. The convergence of the method is treated in the original article along with the illustrative examples that compare the approximation and the exact solutions showing outstanding performance. The following section describes the method adapted to the setting of this article to keep it self-contained. The presentation follows the development in Akyuz-Dascioglu and Sezer (2005).

\subsection{Approximation by Chebyshev polynomials}

In matrix notation the transformed system is given by

$$
\begin{gathered}
\mathbf{P}_{2}(v) \frac{\mathrm{d}^{2}}{\mathrm{~d} v^{2}} \boldsymbol{\Gamma}_{\alpha}(v)+\mathbf{P}_{1}(v) \frac{\mathrm{d}}{\mathrm{d} v} \boldsymbol{\Gamma}_{\alpha}(v)+\mathbf{P}_{0}(v) \boldsymbol{\Gamma}_{\alpha}(v) \\
=\mathbf{g}(v)+\int_{0}^{v} \mathbf{K}(v, y) \boldsymbol{\Gamma}_{\alpha}(y) \mathrm{d} y
\end{gathered}
$$

where $\boldsymbol{\Gamma}_{\alpha}(v)$ is the column vector of unknown functions $\boldsymbol{\Gamma}_{\alpha}(v)=$ $\left(\Gamma_{\alpha}^{1}(v), \Gamma_{\alpha}^{2}(v), \ldots, \Gamma_{\alpha}^{n}(v)\right)^{\top}$. Coefficient matrices are as follows

$$
\begin{aligned}
& \mathbf{P}_{2}(v)=\frac{A(v)}{h^{\prime}(v)} \cdot \mathrm{I}_{n} \\
& \mathbf{P}_{1}(v)=h^{\prime}(v)^{-1} \operatorname{diag}\left(B_{i}(v)\right) \\
& \mathbf{P}_{0}(v)=h^{\prime}(v)^{-1}\left[Q-(\alpha+\lambda) \cdot \mathrm{I}_{n}\right] \\
& \mathbf{K}(v, y)=-\lambda f(h(v)-h(y)) \frac{h^{\prime}(y)}{h^{\prime}(v)} \cdot \mathrm{I}_{n} \\
& \mathbf{g}(v)=-h^{\prime}(v)^{-1}[\alpha P(h(v))+\lambda S(v)] \cdot 1_{n} \\
& S(v)=\int_{v}^{1} \pi(h(v)-h(y)) f(h(y)) h^{\prime}(y) \mathrm{d} y,
\end{aligned}
$$

where $\mathrm{I}_{n}$ is the identity matrix of order $n \times n$ and $1_{n}$ is the column vector of ones of order $n \times 1$. The transform is performed with an arbitrary strictly monotone, twice continuously differentiable function $h:[0,1] \rightarrow[0, \infty)$.

The aim of the method is to approximate the solution by a truncated Chebyshev expansion

$\Gamma_{\alpha}^{i}(v)=\sum_{r=0}^{N} a_{i r}^{*} T_{r}^{*}(v) \quad i=1, \ldots, n$ 
on the interval [0,1], where $T_{r}^{*}(v)$ are shifted Chebyshev polynomials of the first kind (see, for example, Boyd (2001)) and $a_{i r}^{*}$ are the unknown coefficients to be determined. In matrix notation $\Gamma_{\alpha}^{i}(v)=T^{*}(v) A_{i}^{*}$,

where $T^{*}(v)=\left(T_{0}^{*}(v), T_{1}^{*}(v), \ldots, T_{N}^{*}(v)\right)$ is a row vector of shifted Chebyshev polynomials up to degree $N$ and $A_{i}^{*}=$ $\left(a_{i 0}^{*}, a_{i 1}^{*}, \ldots, a_{i N}^{*}\right)^{\top}$ is a column vector of the corresponding coefficients. Similarly, the $n$th derivative of $\Gamma_{\alpha}^{i}(v)$ can be expanded into

$$
\frac{\mathrm{d}^{n}}{\mathrm{~d} v^{n}} \Gamma_{\alpha}^{i}(v)=T^{*}(v) A_{i}^{*(n)}
$$

The link between coefficients $A_{i}^{*(n)}$ and $A_{i}^{*}$ from Sezer and Kaynak (1996) is

$A_{i}^{*(n)}=4^{n} M^{n} A_{i}^{*}$,

where

$$
\begin{aligned}
M & =\left(\begin{array}{cccccccc}
0 & \frac{1}{2} & 0 & \frac{3}{2} & 0 & \frac{5}{2} & \cdots & \frac{N}{2} \\
0 & 0 & 2 & 0 & 4 & 0 & \cdots & 0 \\
0 & 0 & 0 & 3 & 0 & 5 & \cdots & N \\
\vdots & \vdots & \vdots & \vdots & \vdots & \vdots & & \vdots \\
0 & 0 & 0 & 0 & 0 & 0 & \cdots & N \\
0 & 0 & 0 & 0 & 0 & 0 & \cdots & 0
\end{array}\right)_{(N+1) \times(N+1)} \text { for odd } N \\
M & =\left(\begin{array}{cccccccc}
0 & \frac{1}{2} & 0 & \frac{3}{2} & 0 & \frac{5}{2} & \cdots & 0 \\
0 & 0 & 2 & 0 & 4 & 0 & \cdots & N \\
0 & 0 & 0 & 3 & 0 & 5 & \cdots & 0 \\
\vdots & \vdots & \vdots & \vdots & \vdots & \vdots & & \vdots \\
0 & 0 & 0 & 0 & 0 & 0 & \cdots & 0 \\
0 & 0 & 0 & 0 & 0 & 0 & \cdots & N
\end{array}\right)_{(N+1) \times(N+1)} \text { for even } N
\end{aligned}
$$

yields the expansion of the $n$th derivative $\frac{\mathrm{d}^{n}}{\mathrm{~d} v^{n}} \Gamma_{\alpha}^{i}(v)$ in terms of Chebyshev coefficients $A_{i}^{*}$.

On the other hand, functions $K_{i j}(v, y)$ can be expanded in variable $y$ into a Chebyshev series

$K_{i j}(v, y)=\sum_{r=0}^{N} k_{r}^{* i j}(v) T_{r}^{*}(y)$

where the Chebyshev coefficients $k_{r}^{* i j}$ are functions of $v$. Using matrix notation for convenience

$$
K_{i j}(v, y)=k^{* i j}(v) T^{*}(y)^{\top},
$$

where $k^{* i j}$ is the row vector of coefficients determined by Clenshaw-Curtis quadrature; see Clenshaw and Curtis (1960).

Substituting (14)-(16), the $i$ th equation $(i=1, \ldots, n)$ of the system (13) is finally obtained:

$$
\begin{aligned}
& h^{\prime}(v)^{-1} A(v) 16 M^{2} T^{*}(v) A_{i}^{*}+h^{\prime}(v)^{-1} B_{i}(v) 4 M T^{*}(v) A_{i}^{*} \\
& \quad+h^{\prime}(v)^{-1}\left[\sum_{j=1}^{n} q_{i j}-(\alpha+\lambda)\right] T^{*}(v) A_{i}^{*} \\
& =g_{i}(v)-\int_{0}^{v} k^{* i j}(v) T^{*}(y)^{\top} T^{*}(y) A_{i}^{*} \mathrm{~d} y .
\end{aligned}
$$

The matrix of the inner product of Chebyshev polynomials

$$
\begin{aligned}
Z^{*}(v) & =\left(z_{i j}^{*}(v)\right) \equiv \int_{0}^{v} T^{*}(y)^{\top} T^{*}(y) \mathrm{d} y \\
& =\frac{1}{2} \int_{-1}^{2 v-1} T(x)^{\top} T(x) \mathrm{d} x=\frac{1}{2}\left(z_{i j}(2 v-1)\right) \\
& =\frac{1}{2} Z(2 v-1)
\end{aligned}
$$

can be computed as shown in Akyuz-Dascioglu (2007), where

$$
\begin{aligned}
& 2 v^{2}-2 \text { for } i+j=1 \\
& \frac{T_{i+j+1}(v)}{i+j+1}-\frac{T_{i+j-1}(v)}{i+j-1}-\frac{1}{i+j+1}+\frac{1}{i+j-1} \\
& +v^{2}-1 \text { for }|i-j|=1 \\
& z_{i j}(v)=\frac{1}{4}\left\{\frac{T_{i+j+1}(v)}{i+j+1}+\frac{T_{1-i-j}(v)}{1-i-j}+\frac{T_{1+i-j}(v)}{1+i-j}+\frac{T_{1-i+j}(v)}{1-i+j}\right. \\
& +2\left(\frac{1}{1-(i+j)^{2}}+\frac{1}{1-(i-j)^{2}}\right) \text { for even } i+j \\
& \frac{T_{i+j+1}(v)}{i+j+1}+\frac{T_{1-i-j}(v)}{1-i-j}+\frac{T_{1+i-j}(v)}{1+i-j}+\frac{T_{1-i+j}(v)}{1-i+j} \\
& -2\left(\frac{1}{1-(i+j)^{2}}+\frac{1}{1-(i-j)^{2}}\right) \text { for odd } i+j,
\end{aligned}
$$

which yields the system

$$
\begin{gathered}
h^{\prime}(v)^{-1} A(v) 8 M^{2} T^{*}(v) A_{i}^{*}+h^{\prime}(v)^{-1} B_{i}(v) 4 M T^{*}(v) A_{i}^{*} \\
+h^{\prime}(v)^{-1}\left[\sum_{j=1}^{n} q_{i j}-(\alpha+\lambda)\right] T^{*}(v) A_{i}^{*}
\end{gathered}
$$$$
=g_{i}(v)-k^{* i j}(v) Z^{*}(v) A_{i}^{*},
$$

for all $i=1, \ldots, n$. The only unknown values are Chebyshev expansion coefficients $A_{i}^{*}$. The collocation method proposed by the authors fits the solution through the collocation points

$x_{s}=\frac{1}{2}\left(1+\cos \left(\frac{s}{N} \pi\right)\right), \quad s=1,2, \ldots,(N-1)$.

Each of the $N-1$ collocation points $x_{s}$ is substituted into the system (17) and yields $n$ linear equations of unknown variable $A_{i}^{*}$, whence $n(N-1)$ equations are obtained. The boundary conditions (12) for $i=1, \ldots, n$,

$T^{*}(0) A_{i}^{*}=\pi(0-)$

$T^{*}(1) A_{i}^{*}=P(\infty)$,

yield another $2 n$ equations. A linear system of $n(N+1)$ equations is constructed and solved for the Chebyshev coefficients $A_{i}^{*}$. Once the approximation $\widetilde{\Gamma}_{\alpha}^{i}(v)=\sum_{r=0}^{N} a_{i r}^{*} T_{r}^{*}(v)$ is obtained, the relationship between the solution of the transformed and the original system from the Theorem 2.1 yields the approximation of the expected penalty-reward function $\widetilde{\Upsilon}_{\alpha}^{i}(u)=\widetilde{\Gamma}_{\alpha}^{i}\left(h^{-1}(u)\right)$.

\section{Numerical examples}

As mentioned before, $\Upsilon_{t}^{i}(u)$ is the Laplace-Carson transform in time of the expected penalty-reward function in a jump-diffusion process. This function has a probabilistic interpretation as the penalty-reward function in an exponentially killed time horizon $H_{\alpha}$. The ultimate case is also unveiled by a straightforward application of the Tauberian theorem

$$
\begin{aligned}
\lim _{\alpha \rightarrow 0} \Upsilon_{\alpha}^{i} & =\lim _{\alpha \rightarrow 0} \int_{0}^{\infty} \alpha \mathrm{e}^{-\alpha t} \phi_{t}^{i}(u) \mathrm{d} t \\
& =-\phi_{0}^{i}(u)+\lim _{\alpha \rightarrow 0} \int_{0}^{\infty} \mathrm{e}^{-\alpha t} \frac{\mathrm{d}}{\mathrm{d} t} \phi_{t}^{i}(u) \mathrm{d} t \\
& =-\phi_{0}^{i}(u)+\int_{0}^{\infty} \frac{\mathrm{d}}{\mathrm{d} t} \phi_{t}^{i}(u) \mathrm{d} t=\phi_{\infty}^{i}(u) .
\end{aligned}
$$

For the more challenging finite time horizon penalty-reward, a numerical inversion of the Laplace transform recovers the original function $\phi_{\alpha}^{i}(u)$; see Usabel (1999). The relationship $C(s)=$ $s L(s)$ between the Laplace transform $L(s)$ and the Laplace-Carson transform $C(s)$ applies. 
Table 1

Ultimate survival probability for gamma distributed claims.

\begin{tabular}{|c|c|c|c|c|c|c|c|}
\hline & & \multicolumn{6}{|c|}{$N$-precision level } \\
\hline & & 200 & 250 & 300 & 350 & 400 & 450 \\
\hline \multirow{5}{*}{$u$} & 1 & 0.318081594 & 0.318079845 & 0.318079373 & 0.318079219 & 0.318079161 & 0.318079137 \\
\hline & 2 & 0.435631392 & 0.43562899 & 0.435628343 & 0.435628132 & 0.435628053 & 0.43562802 \\
\hline & 5 & 0.753759689 & 0.753755453 & 0.753754322 & 0.753753953 & 0.753753813 & 0.753753756 \\
\hline & 10 & 0.987580029 & 0.987573342 & 0.987571486 & 0.98757086 & 0.987570616 & 0.987570511 \\
\hline & 15 & 0.999982762 & 0.999973643 & 0.99997087 & 0.999969864 & 0.999969447 & 0.999969256 \\
\hline
\end{tabular}

Table 2

Survival probability under Markov-modulated interest rate structure.

\begin{tabular}{|c|c|c|c|c|c|c|}
\hline \multirow[t]{2}{*}{$u$} & \multirow[t]{2}{*}{$\delta_{i}(\%)$} & \multicolumn{5}{|c|}{$N$-precision level } \\
\hline & & 250 & 300 & 350 & 400 & 450 \\
\hline \multirow{5}{*}{1} & 1 & 0.144815222 & 0.144815829 & 0.144815469 & 0.144814893 & 0.144814330 \\
\hline & 2 & 0.146306443 & 0.146307016 & 0.146306644 & 0.146306063 & 0.146305499 \\
\hline & 7 & 0.188906830 & 0.188906028 & 0.188905174 & 0.188904470 & 0.188903928 \\
\hline & 8 & 0.190954404 & 0.190953560 & 0.190952690 & 0.190951981 & 0.190951439 \\
\hline & 9 & 0.191794388 & 0.191793534 & 0.191792659 & 0.191791949 & 0.191791406 \\
\hline \multirow{5}{*}{10} & 1 & 0.676382452 & 0.676390197 & 0.676389970 & 0.676387433 & 0.676384493 \\
\hline & 2 & 0.689328522 & 0.689335954 & 0.689335662 & 0.689333147 & 0.689330254 \\
\hline & 7 & 0.855051985 & 0.855051380 & 0.855048719 & 0.855045981 & 0.855043652 \\
\hline & 8 & 0.865060563 & 0.865059778 & 0.865057124 & 0.865054446 & 0.865052186 \\
\hline & 9 & 0.870653629 & 0.870652847 & 0.870650244 & 0.870647620 & 0.870645408 \\
\hline \multirow{5}{*}{15} & 1 & 0.845057051 & 0.845074744 & 0.845078953 & 0.845078338 & 0.845076134 \\
\hline & 2 & 0.864897819 & 0.864914116 & 0.864918001 & 0.864917442 & 0.864915420 \\
\hline & 7 & 0.977203365 & 0.977208141 & 0.977208683 & 0.977207860 & 0.977206693 \\
\hline & 8 & 0.981995614 & 0.981999965 & 0.982000462 & 0.981999717 & 0.981998658 \\
\hline & 9 & 0.984633935 & 0.984638092 & 0.984638607 & 0.984637937 & 0.984636962 \\
\hline \multirow{5}{*}{20} & 1 & 0.949938439 & 0.949967119 & 0.949978339 & 0.949982157 & 0.949982771 \\
\hline & 2 & 0.967826609 & 0.967849612 & 0.967858765 & 0.967861995 & 0.967862632 \\
\hline & 7 & 0.999402042 & 0.999408967 & 0.999411851 & 0.999412960 & 0.999413267 \\
\hline & 8 & 0.999715443 & 0.999721564 & 0.999724130 & 0.999725128 & 0.999725416 \\
\hline & 9 & 0.999823318 & 0.999828873 & 0.999831216 & 0.999832138 & 0.999832412 \\
\hline \multirow{5}{*}{25} & 1 & 0.993079369 & 0.993110103 & 0.993125249 & 0.993132811 & 0.993136514 \\
\hline & 2 & 0.998186486 & 0.998205547 & 0.998215150 & 0.998220073 & 0.998222575 \\
\hline & 7 & 0.999986074 & 0.999991170 & 0.999993799 & 0.999995183 & 0.999995911 \\
\hline & 8 & 0.999988832 & 0.999993290 & 0.999995592 & 0.999996805 & 0.999997445 \\
\hline & 9 & 0.999990114 & 0.999994091 & 0.999996147 & 0.999997232 & 0.999997804 \\
\hline
\end{tabular}

\subsection{Ultimate survival probability}

The survival probability is a special case of the function $\Upsilon_{\alpha}^{i}(u)$. For $\pi(x) \equiv 0$ and $P(x) \equiv 1$

$\phi_{\infty}^{i}(u)=\mathrm{E}\left[\mathbb{I}(\tau=\infty) \mid U_{0}=u, \Delta_{0}=\delta_{i}\right]$.

The premium collection rate is $c=11$, the volatility of premium accruals $\sigma^{2}=0.04$, the intensity of claim arrivals $\lambda=4$, and claims follow a Gamma distribution $\operatorname{Gamma}(5 ; 2)$. The interest rate is assumed to be fixed at $3 \%$ with no volatility $\left(\sigma_{r}^{2}=0\right)$. The ultimate survival probability $\phi_{\infty}^{i}(u)$ is considered in this context and thus $\alpha=0$ as motivated by (18). For the change of variables, the function $h(v)=-\ln (1-v)$ was used. The Table 1 shows the approximations for various starting reserves and precision levels (order of Chebyshev polynomials).

\subsection{Markov-modulated interest rate structure}

The second example presents an interest rate structure driven by a Markov process and a reserve dependent volatility. Let us assume two regimes (high interest rate and low interest rate) comprising several interest rate levels. The intensity matrix $Q$, characterizing the Markov process, governs the evolution of the interest rate:

$$
\left.Q=\begin{array}{cccccc}
\delta_{i} & 1 \% & 2 \% & 7 \% & 8 \% & 9 \% \\
1 \% & 2 \% \\
7 \% & -2 & 2 & 0 & 0 & 0 \\
8 \% & 1.9 & -2 & 0.1 & 0 & 0 \\
9 \% & 0 & 0.1 & -3 & 2.9 & 0 \\
0 & 0 & 1 & -3 & 2 \\
0 & 0 & 0 & 3 & -3
\end{array}\right) .
$$

The low interest rate regime embeds two levels $1 \%$ and $2 \%$ while the high interest rate regime considers three levels $7 \%, 8 \%$, and $9 \%$. Let the premium collection rate be 1 with the volatility of premium accruals 0.25 , the intensity of claims arrival $\frac{1}{3}$ (one claim every three time periods on average), the distribution of claim size lognormal $\ln \mathcal{N}(0.5 ; 1)$. The volatility of the return on investment, dependent on the reserves level, is $\kappa^{2}(u)=\frac{\sigma_{r}^{2}}{u}$, as motivated in the introduction, with $\sigma_{r}^{2}=0.81$. The probability of survival of a random horizon of 20 years on average is approximated $(\alpha=0.05$, $\pi(x) \equiv 0$ and $P(x) \equiv 1)$. Regarding the change of variables, the function $h(v)=-\ln (1-v)$ was used again. In Table 2 the survival probabilities conditional on various initial interest rates and starting reserve levels are presented.

Fig. 1 unveils the impact of the initial conditions on the survival probability. Each curve represents different initial interest rate, the lowest curve corresponds to $\Delta_{0}=1 \%$ and the uppermost to $\Delta_{0}=9 \%$. The horizontal axis shows the initial reserves level $U_{0}$, the vertical axis the survival probability $\Upsilon_{\alpha}^{i}(u)$.

\section{Conclusions}

A general model for the risk process of an insurance company is presented allowing arbitrary distributions of the claim sizes, a Wiener fluctuation in premium collection and investment in a, possibly, risky asset. The evolution of the return rate is modulated by Markov process implementing a non-constant interest rates in a risk process. In particular, we suggest the possibility of interpretation as interest rates announced by a central bank that in 


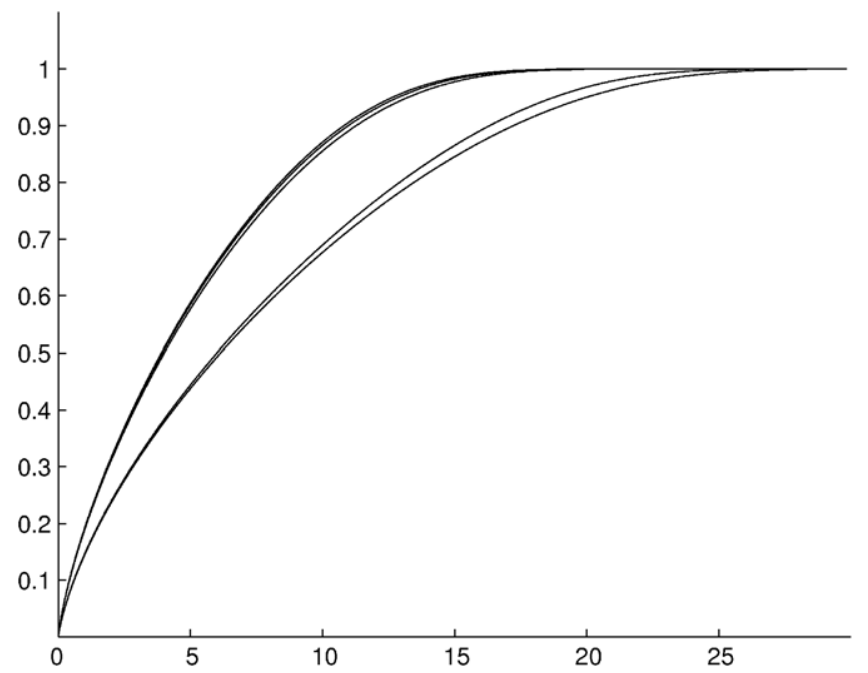

Fig. 1. Survival probability curves as a function of initial reserves. Each curve represents different initial interest level, the lowest curve corresponding to $1 \%$, the uppermost to $9 \%$.

practice move by a quarter percentile jumps. A method is obtained to calculate the Gerber-Shiu expected penalty-reward function in this framework that comprises several interesting particular cases such as the calculation of ruin probabilities or moments of the deficit at ruin. The method is based on Chebyshev polynomial approximations and shows an outstanding convergence rate.

\section{Acknowledgements}

The authors gratefully acknowledge the financial support of the PWC Professorship at UC3M.

\section{References}

Akyuz-Dascioglu, A., 2007. A Chebyshev polynomial approach for linear Fredholm-Volterra integro-differential equations in the most general form. Applied Mathematics and Computation 181 (1), 103-112.

Akyuz-Dascioglu, A., Sezer, M., 2005. Chebyshev polynomial solutions of systems of higher-order linear Fredholm-Volterra integro-differential equations. Journal of the Franklin Institute 342 (6), 688-701.

Asmussen, S., Albrecher, H., 2010. Ruin Probabilities. World Scientific, Singapore.

Avram, F., Usabel, M., 2008. The Gerber-Shiu expected discounted penalty-reward function under an affine jump-diffusion model. Astin Bulletin 38 (2), 461-481.

Berk, J., Green, R., 2004. Mutual fund flows and performance in rational markets. Journal of Political Economy 112 (6), 1269-1295.
Boyd, J., 2001. Chebyshev and Fourier Spectral Methods, 2nd ed. Dover, New York. Cai, J., Yang, H., 2005. Ruin in the perturbed compound Poisson risk process under interest force. Advances in Applied Probability 37 (3), 819-835.

Clenshaw, C., Curtis, A., 1960. A method for numerical integration on an automatic computer. Numerische Mathematik 2 (1), 197-205.

Dufresne, F., Gerber, H., 1991. Risk theory for the compound Poisson process that is perturbed by diffusion. Insurance Mathematics and Economics 10 (1), 51-59.

Gaier, J., Grandits, P., 2004. Ruin probabilities and investment under interest force in the presence of regularly varying tails. Scandinavian Actuarial Journal 2004 (4), 256-278

Gerber, H., 1970. An extension of the renewal equation and its application in the collective theory of risk. Skandinavisk Aktuarietidskrift 53, 205-210.

Gerber, H., Landry, B., 1998. On the discounted penalty at ruin in a jump-diffusion and the perpetual put option. Insurance Mathematics and Economics 22 (3), 263-276.

Gerber, H., Shiu, E., 1997. The joint distribution of the time of ruin, the surplus immediately before ruin, and the deficit at ruin. Insurance: Mathematics and Economics 21 (2), 129-137.

Gerber, H., Shiu, E., 1998. On the time value of ruin. North American Actuarial Journal 2, 48-71.

Grandits, P., 2005. Minimal ruin probabilities and investment under interest force for a class of subexponential distributions. Scandinavian Actuarial Journal 2005 (6), 401-416.

Kushner, H.J.J., Dupuis, P., 1992. Numerical Methods for Stochastic Control Problems in Continuous Time. Springer-Verlag, New York.

Le, U., Pascali, E., 2009. Existence theorems for systems of nonlinear integro-differential equations. Ricerche di matematica 58 (1), 91-101.

Li, S., Garrido, J., 2005. Ruin probabilities for two classes of risk processes. ASTIN Bulletin 35 (1), 61-77.

Ma, J., Sun, X., 2003. Ruin probabilities for insurance models involving investments. Scandinavian actuarial journal 2003 (3), 217-237.

Morales, M., 2007. On the expected discounted penalty function for a perturbed risk process driven by a subordinator. Insurance Mathematics and Economics 40 (2), 293-301.

Paulsen, J., 1993. Risk theory in a stochastic economic environment. Stochastic Processes and their Applications 46 (2), 327-361.

Paulsen, J., Gjessing, H., 1997. Optimal choice of dividend barriers for a risk process with stochastic return on investments. Insurance Mathematics and Economics 20 (3), 215-223.

Ren, J., 2005. The expected value of the time of ruin and the moments of the discounted deficit at ruin in the perturbed classical risk process. Insurance Mathematics and Economics 37 (3), 505-521.

Risken, H., 1996. The Fokker-Planck Equation: Methods of Solution and Applications. Springer Verlag, Berlin.

Sarkar, J., Sen, A., 2005. Weak convergence approach to compound Poisson risk processes perturbed by diffusion. Insurance Mathematics and Economics 36 (3), 421-432.

Sezer, M., Kaynak, M., 1996. Chebyshev polynomial solutions of linear differential equations. International Journal of Mathematical Education in Science and Technology 27 (4), 607-618.

Usabel, M., 1999. Calculating multivariate ruin probabilities via Gaver-Stehfest inversion technique. Insurance: Mathematics and Economics 25 (2), 133-142.

Wang, G., 2001. A decomposition of the ruin probability for the risk process perturbed by diffusion. Insurance Mathematics and Economics 28 (1), 49-59.

Wang, G., Wu, R., 2008. The expected discounted penalty function for the perturbed compound Poisson risk process with constant interest. Insurance: Mathematics and Economics 42 (1), 59-64. 